Curr Opin Insect Sci. 2015 February 1; 7: 82-86. doi:10.1016/j.cois.2015.03.001.

\title{
Ageing and Circadian rhythms
}

\author{
Jadwiga M. Giebultowicz and Dani M. Long \\ Department of Integrative Biology, Oregon State University, Corvallis, OR, USA
}

\begin{abstract}
Circadian clocks are cell-autonomous molecular feedback loops that generate daily rhythms in gene expression, cellular functions, physiological processes and behavior. The mechanisms of circadian clocks are well understood in young fruit flies Drosophila melanogaster, but less is known about how circadian system changes during organismal aging. Similar as in humans, rest/ activity rhythms tend to weaken with age in fruit flies, suggesting conservation of aging-related changes in the circadian system. It has been shown that aging is associated with reduced expression of core clock genes in peripheral head clocks while similar reduction may not occur in central clock neurons regulating behavioral rhythms. Arrhythmic flies with mutations in core clock genes display accelerated aging and shortened lifespan suggesting that weakened circadian rhythms may contribute to aging phenotypes. To understand whether strong circadian clocks support organism's healthspan and lifespan, future research needs to focus on age-related changes in clock genes as well as clock-controlled genes in specific organs and tissues.
\end{abstract}

Circadian clocks are cell-autonomous molecular feedback loops that generate daily rhythms in gene expression, cellular functions, physiological processes and behavior. Among insects, the clock mechanism is best understood in Drosophila melanogaster (see article by Tataroglu and Emery in this issue). Briefly, two transcription factors encoded by the genes Clock (Clk) and cycle (cyc) stimulate the transcription of period (per) and timeless (tim) genes in the early night. PER and TIM proteins accumulate in the cell nuclei and repress CLK-CYC activity, resulting in the suppression of per and tim transcription [1]. Clock oscillations are enhanced by many other genes, including Par domain protein $1 \varepsilon(P d p 1 \varepsilon)$ and vrille (vri) [1]. Circadian rhythms persist in experimental conditions of constant darkness (DD) with a circa $24 \mathrm{~h}$ period, but are normally entrained to daily light/dark (LD) cycles via light-sensitive CRY protein encoded by the cryptochrome (cry) gene. The fly circadian system consists of the central clock neurons, which control rest/activity rhythms, and a multitude of peripheral clocks in sensory neurons, glia, fat bodies, gut and excretory epithelia. Peripheral clocks function independently of the central clock, but they cycle less robustly in constant conditions [2,3]. In contrast to the central clock, the output rhythms of many peripheral clocks are not well understood.

(C) 2015 Published by Elsevier Inc.

*Corresponding author: Jadwiga M. Giebultowicz, Oregon State University, Department of Integrative Biology, 3029 Cordley Hall, Corvallis, OR 97331 USA, Phone: (541) 737-5530, giebultj@ science.oregonstate.edu.

Publisher's Disclaimer: This is a PDF file of an unedited manuscript that has been accepted for publication. As a service to our customers we are providing this early version of the manuscript. The manuscript will undergo copyediting, typesetting, and review of the resulting proof before it is published in its final citable form. Please note that during the production process errors may be discovered which could affect the content, and all legal disclaimers that apply to the journal pertain. 
Aging is associated with a loss of temporal coordination in vital functions. A well-known symptom of old age in mammals is the weakening of rhythmic behaviors, such as circadian sleep/wake rhythms [4]. Rhythmic behaviors were not investigated across lifespan in insects until a pioneer study that revealed that decline in sleep consolidation and weakened rest/ activity rhythms occur also in aging fruit flies Drosophila [5]. This work opened the road to study the reciprocal relationship between aging and the circadian system in insects. Due to their short lifespan (in the range of 50-80 days) and excellent genetic tools at hand, flies may help to determine whether the decay of circadian rhythms with age is just a biomarker of senescence or - more interestingly - whether there is a causative relationship between weakened circadian rhythms and aging. The goal of this review is to summarize modest inroads that were so far made in this area of research and to illuminate the power of insects for future studies on aging circadian system.

\section{Aging alters activity rhythms and circadian clockwork in D. melanogaster}

Young flies have long consolidated bouts of sleep while aging is associated with more frequent and shorter sleep bouts and declining strength of the overall rest/activity rhythm [5]. Subsequent studies provided further evidence for age-related decay of rest/activity rhythms (Figure 1), such as the lengthening of the free running circadian period and an increasing percentage of flies becoming weakly rhythmic or arrhythmic [6-9]. Very old flies showed drifts in the length of free running period with females exhibiting increased incidences of phase instability and arrhythmicity in constant darkness compared to males of the same age [6].

Breakdown in behavioral rhythms is accompanied by changes in clock gene expression in peripheral clocks in fly heads. Clock genes per and tim and their proteins showed dampened oscillations in heads of old flies compared to young, and similar changes were detected in clock-associated genes $P d p 1 \varepsilon$, vri, and cry [6,7]. Decreased clock oscillations in heads suggest that peripheral clocks are affected, as they form a bulk of head clocks. Indeed, it was determined by immunocytochemistry that retinal photoreceptor cells in the compound eyes of old flies have reduced expression of nuclear PER at the expected peak compared to young flies [6,7]. While retinal peripheral clocks were impaired by age, this was not the case for Malpighian tubules as strong PER oscillations were observed in 50-days old flies. With regard to central clock neurons, it was demonstrated that they maintain strong PER rhythm in one study [6], while another study reported reduced PER and TIM oscillations in these neurons in old flies [9]. These differences may reflect the fact that aging rate and lifespan can show substantial differences dependent on genetic background [5]. Another age-related change observed in the central clock network was reduction in the levels of Pigment Dispersing Factor (PDF) [6,9], a neuropeptide that helps to synchronize clock oscillation in central clock neurons of young flies [10].

An important question that emerged from the studies discussed above is whether reduced expression of clock genes is causally linked to the decay of rest/activity rhythms. If so, then overexpression of genes reduced by aging should prevent decay of rest/activity rhythms. Indeed, overexpression of PDF in PDF-positive neurons partially rescued behavioral 
rhythms and shortened free-running periods in old flies, and also increased TIM (but not PER) expression in specific central clock neurons [9].

In addition to PDF, aging causes significant reduction in the level of CRY protein, which mediates entrainment of clocks to light and is also involved in peripheral clock function in their free-running mode in constant darkness [11]. Overexpression of cry in all clock cells (using tim-Gal4 driver) significantly enhanced expression of several clock genes and strengthened rest/activity rhythms in old flies as well as delayed their physiological aging [11]. Interestingly, overexpression of cry in central clock neurons alone was not sufficient to restore rest/activity rhythms suggesting that peripheral clocks play an active role in delaying behavioral and physiological aging [11]. The rest/activity rhythms were also enhanced in old flies by coupling light-dark cycles (LD) with a high-low temperature cycles [6]. Taken together these studies suggest that age-related decline in rest/activity rhythm can be reversed by genetic or environmental manipulations. They also suggest that aging may weaken behavioral rhythms downstream of central clock, which can maintain strong oscillations until very advanced age [6].

\section{Effects of clock gene mutants on lifespan and healthspan}

Physiological aging is accelerated by chronic disruption of clock functions in mammals, and a null mutation in the core clock gene Bmall (homolog of cyc) significantly shorten lifespan in mice $[12,13]$. It appears that longevity is also affected in flies with mutations in core clock genes. Shortened lifespan was reported in per mutants with altered free-running period [14], and in cyc-null males (but not females) [15]. In addition to lifespan, the healthspan of flies may be affected by disruption of circadian clocks. When middle-age per-null mutants were exposed to $24-\mathrm{h}$ hyperoxic stress (100\% oxygen), their mortality rate significantly increased compared to age-matched wild-type flies exposed to this stress [16]. Further, pernull mutant flies had significantly higher accumulation of oxidatively damaged proteins and lipids along with accelerated loss of vertical climbing ability [16]. Poor climbing ability in per-null flies was associated with increased neurodegeneration in 50-days old flies [16], suggesting that clocks may have neuroprotective functions during aging. This was further supported when lifespan was tested in neurodegeneration-prone sniffer (sni) [17] and swiss cheese (sws) [18] mutants in per-positive and per-null backgrounds. sni or sws mutants with disrupted clocks exhibited shortened lifespan and more severe neurodegeneration at a younger age compared to either sni or sws single mutant with normal clock function [19]. Together, these results suggest that possessing a functional circadian clock may play neuroprotective roles during aging by coordinating temporal homeostasis in the aging brain.

The neuroprotective role of the circadian clock may not apply to exogenous pathological factors introduced into Drosophila disease models. When human amyloid $\beta$ (A $\beta)$ peptides were expressed in flies to create Alzheimer disease (AD) models, disruption of a functional circadian system via per-null mutation had little effect on fly lifespan or healthspan [20]. On the other hand, overexpression of pathogenic $\mathrm{A} \beta 42$ peptide carrying the arctic mutation (A $\beta 42 \mathrm{arc})$ led to accelerated decline in rest/activity rhythms, combined with loss of vertical climbing ability and increased neurodegeneration even in 15-20 days old flies [20,21]. Surprisingly, central clock neurons of these flies were intact and exhibited robust cycling in 
PER with a peak and trough that was not significantly different from age matched controls $[20,21]$. These studies suggest that damage to clock output pathways downstream of the central clock could be responsible for the loss of rest/activity rhythms in AD model flies. As pathways leading from central clock to rhythmic behavior are being identified [22] flies may serve as an excellent model to investigate often observed but poorly understood links between neurodegenerative diseases and loss of sleep/wake rhythms in humans [23].

\section{What are the bases of anti-aging effects of the functional circadian system?}

The emerging evidence linking the circadian system with delayed aging calls for the understanding of the pathways mediating these relationships. Circadian clocks impose rhythmicity on the expression of many target genes, so called clock-controlled genes (CCGs). Genome-wide studies of the circadian transcriptome determined that many CCGs act in healthspan supporting pathways regulating redox, ROS homeostasis, and detoxification in central and peripheral clocks [24-26]. Yet, it is not known how aging affects expression of these CCGs on a genome wide-scale. Among CCGs that are rhythmically expressed in heads of young flies are Gclc and Gclm genes which are involved in biosynthesis of a major endogenous antioxidant, glutathione [27]. We recently found that the expression of these genes is arrhythmic in heads of old flies (Klichko et al, in preparation). It could be hypothesized that aging of the circadian clock may lead to dampened rhythms or arrhythmicity in many CCGs maintaining temporal homeostasis in the nervous system of young flies (Figure 1). Deregulation of temporal homeostasis in the aging brain could, in turn, accelerate age related pathologies such as neurodegeneration, and thus weaken rest/activity rhythms. This hypothesis could be tested by investigating the effects of aging on circadian regulation on a genome-wide scale in Drosophila, which is an excellent model in both circadian and aging studies.

\section{Interactions between clocks, endocrine signaling, and longevity}

Aging and longevity are regulated by insulin/insulin-like growth factor (IGF) signaling (IIS), which is activated by nutrient intake. Reduced IIS extends healthy lifespan in many animals including Drosophila. There are many reciprocal links between IIS and the circadian system in young Drosophila. First, some genes in this pathway are expressed rhythmically in heads of young flies [28]. Secondly, manipulation of the AKT and TOR-S6K pathways, which are major regulators of nutrient metabolism, cell growth, and senescence, impacts the free running period of the central circadian clock that drives behavioral rhythms in Drosophila [29]. Finally, reduced TOR or insulin signaling can reverse age-related sleep decline in flies [30]. It would be interesting to test whether reduced IIS signaling improves the circadian rhythm of clock gene expression in aging flies.

Insulin signaling is also involved in insect reproductive diapause which is a prominent way to extend female lifespan. Diapause is a programmed arrested state of development that allows insects to survive adverse seasonal conditions. Cessation of reproduction occurs in response to inhibition of juvenile hormone $(\mathrm{JH})$, but studies in a number of insects suggest that lifespan is shortened by increased JH and extended by the removal of JH-producing glands independently of reproduction [31-33]. Interestingly, some genes in the JH 
biosynthetic pathway show circadian rhythmicity in the fruit fly, mosquito, and honey bee [34], and additionally high amplitude rhythms in hemolymph JH levels were reported in the migratory but not in the reproducing cricket morph [35]. However, it remains to be determined whether rhythms in JH biosynthesis exist in adult insects of other species and, if so, whether they affect aging and lifespan of insects.

There is increasing evidence that health status of single tissue types may affect insect longevity. Specifically, regenerative potential of the intestinal stem cells (ISC) affects aging in D. melanogaster [36]. Unexpectedly, the core clock gene period, was reported to be critical for synchronizing ISC cell divisions during regeneration of damaged gut epithelium [37], suggesting that the clock may be important for long-term tissue homeostasis and the ability of stem cells to self-renew. However, with the exception of a few tissues (central clock neurons, retinal photoreceptors, and Malpighian tubules) we do not know how the expression of clock genes and CCGs change with age in other cells with functional clocks.

\section{Conclusions and future directions}

Research on the relationships between circadian clocks and aging is in an early exploratory phase with more questions than answers. The status of central clock neurons in old flies seems to require further research, but it is clear that rest/activity rhythms can become disrupted by normal aging or by the introduction of pathogenic $\mathrm{A} \beta$ peptides despite strong oscillations of clock genes in the central clock neurons [6,20,21]. It is possible that peripheral clocks in the fly head, while dispensable for strong rest/activity rhythms in young flies, may be important in old flies via their role in maintaining temporal homeostasis in the nervous system. To address these questions, future research needs to focus on age-related changes in clock genes as well as clock-controlled genes and processes in specific tissues.

\section{Acknowledgments}

We thank Drs. Christine Merlin and Guy Bloch for helpful input, and Eileen Chow, Rachael Kuintzle, and David Hendrix for reading the manuscript. Author's research reported in this publication was supported by the National Institute of Aging of the National Institutes of Health under award number R01 AG045830 to JMG.

\section{References}

1. Hardin PE. Molecular genetic analysis of circadian timekeeping in Drosophila. Adv Genet. 2011; 74:141-173. [PubMed: 21924977]

2. Giebultowicz JM. Peripheral clocks and their role in circadian timing: insights from insects. Phil Trans R Soc B. 2001; 356:1791-1799. [PubMed: 11710986]

3. Tomioka K, Uryu O, Kamae Y, Umezaki Y, Yoshii T. Peripheral circadian rhythms and their regulatory mechanism in insects and some other arthropods: a review. J Comp Physiol B. 2012; 182:729-740. [PubMed: 22327195]

4. Kondratova AA, Kondratov RV. The circadian clock and pathology of the ageing brain. Nat Rev Neurosci. 2012; 13:325-335. [PubMed: 22395806]

5. Koh K, Evans JM, Hendricks JC, Sehgal A. A Drosophila model for age-associated changes in sleep:wake cycles. Proc Natl Acad Sci U S A. 2006; 103:13843-13847. [PubMed: 16938867]

6•. Luo W, Chen WF, Yue Z, Chen D, Sowcik M, Sehgal A, Zheng X. Old flies have a robust central oscillator but weaker behavioral rhythms that can be improved by genetic and environmental manipulations. Aging Cell. 2012; 11:428-438. This study determined that aging alters rhythmic behavior despite strong oscillations in central clock neurons in old animals. [PubMed: 22268765] 
7. Rakshit K, Krishnan N, Guzik EM, Pyza E, Giebultowicz JM. Effects of aging on the molecular circadian oscillations in Drosophila. Chronobiol Int. 2012; 29:1-10. [PubMed: 22217095]

8. Rezaval C, Berni J, Gorostiza EA, Werbajh S, Fagilde MM, Fernandez MP, Beckwith EJ, Aranovich EJ, Sabio y Garcia CA, Ceriani MF. A functional misexpression screen uncovers a role for enabled in progressive neurodegeneration. PLoS One. 2008; 3:e3332. [PubMed: 18841196]

9•. Umezaki Y, Yoshii T, Kawaguchi T, Helfrich-Forster C, Tomioka K. Pigment-dispersing factor is involved in age-dependent rhythm changes in Drosophila melanogaster. J Biol Rhythms. 2012; 27:423-432. This study established that neuropeptide that synchonize central clock neurons decline with age and its overexpression can rejuvenate rest/activity rhythms in old flies. [PubMed: 23223368]

10. Yoshii T, Wulbeck C, Sehadova H, Veleri S, Bichler D, Stanewsky R, Helfrich-Forster C. The neuropeptide pigment-dispersing factor adjusts period and phase of Drosophila's clock. J Neurosci. 2009; 29:2597-2610. [PubMed: 19244536]

11. Rakshit K, Giebultowicz JM. Cryptochrome restores dampened circadian rhythms and promotes healthspan in aging Drosophila. Aging Cell. 2013; 12:752-762. This study determined that cryptochrome is reduced in old flies at mRNA and protein levels. Overexpression of this gene in all clock cells not only improve rest/activity rhythm but also restore climbing ability and reduce oxidative damage in old flies. [PubMed: 23692507]

12. Kondratov RV, Kondratova AA, Gorbacheva VY, Vykhovanets OV, Antoch MP. Early aging and age-related pathologies in mice deficient in BMAL1, the core component of the circadian clock. Genes Dev. 2006; 20:1868-1873. [PubMed: 16847346]

13. Yu EA, Weaver DR. Disrupting the circadian clock: gene-specific effects on aging, cancer, and other phenotypes. Aging (Albany NY). 2011; 3:479-493. [PubMed: 21566258]

14. Klarsfeld A, Rouyer F. Effects of circadian mutations and LD periodicity on the life span of Drosophila melanogaster. J Biol Rhythms. 1998; 13:471-478. [PubMed: 9850008]

15. Hendricks JC, Lu S, Kume K, Yin JC, Yang Z, Sehgal A. Gender dimorphism in the role of cycle (BMAL1) in rest, rest regulation, and longevity in Drosophila melanogaster. J Biol Rhythms. 2003; 18:12-25. [PubMed: 12568241]

16. Krishnan N, Kretzschmar D, Rakshit K, Chow E, Giebultowicz J. The circadian clock gene period extends healthspan in aging Drosophila melanogaster. Aging. 2009; 1:937-948. [PubMed: 20157575]

17. Botella JA, Ulschmid JK, Gruenewald C, Moehle C, Kretzschmar D, Becker K, Schneuwly S. The Drosophila carbonyl reductase sniffer prevents oxidative stress-induced neurodegeneration. Curr Biol. 2004; 14:782-786. [PubMed: 15120069]

18. Kretzschmar D, Hasan G, Sharma S, Heisenberg M, Benzer S. The swiss cheese mutant causes glial hyperwrapping and brain degeneration in Drosophila. J Neurosci. 1997; 17:7425-7432. [PubMed: 9295388]

19•. Krishnan N, Rakshit K, Chow ES, Wentzell JS, Kretzschmar D, Giebultowicz JM. Loss of circadian clock accelerates aging in neurodegeneration-prone mutants. Neurobiol Dis. 2012; 45:1129-1135. This work demonstrated that mutation in clock gene period dramatically accelerates aging in neurodegeneration-prone fly mutants. [PubMed: 22227001]

20. Long DM, Blake MR, Dutta S, Holbrook SD, Kotwica-Rolinska J, Kretzschmar D, Giebultowicz JM. Relationships between the circadian system and Alzheimer's disease-like symptoms in Drosophila. PLoS One. 2014; 9:e106068. [PubMed: 25171136]

21. Chen KF, Possidente B, Lomas DA, Crowther DC. The central molecular clock is robust in the face of behavioural arrhythmia in a Drosophila model of Alzheimer's disease. Dis Model Mech. 2014; 7:445-458. [PubMed: 24574361]

22. Cavanaugh DJ, Geratowski JD, Wooltorton JR, Spaethling JM, Hector CE, Zheng X, Johnson EC, Eberwine JH, Sehgal A. Identification of a circadian output circuit for rest:activity rhythms in Drosophila. Cell. 2014; 157:689-701. [PubMed: 24766812]

23. Hastings MH, Goedert M. Circadian clocks and neurodegenerative diseases: time to aggregate? Current Opinion in Neurobiology. 2013

24. Wijnen H, Young MW. Interplay of circadian clocks and metabolic rhythms. Annu Rev Genet. 2006; 40:409-448. [PubMed: 17094740] 
25. Hooven LA, Sherman KA, Butcher S, Giebultowicz JM. Does the clock make the poison? Circadian variation in response to pesticides. PLoS One. 2009; 4:e6469. [PubMed: 19649249]

26. Xu K, DiAngelo JR, Hughes ME, Hogenesch JB, Sehgal A. The circadian clock interacts with metabolic physiology to influence reproductive fitness. Cell Metab. 2011; 13:639-654. [PubMed: 21641546]

27. Beaver LM, Klichko VI, Chow ES, Kotwica-Rolinska J, Williamson M, Orr WC, Radyuk SN, Giebultowicz JM. Circadian regulation of glutathione levels and biosynthesis in Drosophila melanogaster. PLoS One. 2012; 7:e50454. [PubMed: 23226288]

28. Sim C, Denlinger DL. Insulin signaling and the regulation of insect diapause. Front Physiol. 2013; 4:189. [PubMed: 23885240]

29. Zheng X, Sehgal A. AKT and TOR signaling set the pace of the circadian pacemaker. Curr Biol. 2010; 20:1203-1208. [PubMed: 20619819]

30. Metaxakis A, Tain LS, Gronke S, Hendrich O, Hinze Y, Birras U, Partridge L. Lowered insulin signalling ameliorates age-related sleep fragmentation in Drosophila. PLoS Biol. 2014; 12:e1001824. [PubMed: 24690889]

31. Herman WS, Tatar M. Juvenile hormone regulation of longevity in the migratory monarch butterfly. Proc Biol Sci. 2001; 268:2509-2514. [PubMed: 11749703]

32. Hodkova M, Okuda T, Wagner RM. Regulation of corpora allata in females of Pyrrhocoris apterus (Heteroptera) (a mini-review). In Vitro Cell Dev Biol Anim. 2001; 37:560-563. [PubMed: 11710430]

33. Yamamoto R, Bai H, Dolezal AG, Amdam G, Tatar M. Juvenile hormone regulation of Drosophila aging. BMC Biol. 2013; 11:85. [PubMed: 23866071]

34. Bloch G, Hazan E, Rafaeli A. Circadian rhythms and endocrine functions in adult insects. J Insect Physiol. 2012

35. Zhao Z, Zera AJ. The hemolymph JH titer exhibits a large-amplitude, morph-dependent, diurnal cycle in the wing-polymorphic cricket, Gryllus firmus. J Insect Physiol. 2004; 50:93-102. [PubMed: 15037097]

36. Wang L, Karpac J, Jasper H. Promoting longevity by maintaining metabolic and proliferative homeostasis. J Exp Biol. 2014; 217:109-118. [PubMed: 24353210]

37. Karpowicz P, Zhang Y, Hogenesch JB, Emery P, Perrimon N. The circadian clock gates the intestinal stem cell regenerative state. Cell Rep. 2013; 3:996-1004. [PubMed: 23583176] 


\section{Highlights}

- Circadian clocks are well understood in young fruit flies but less is known about how they change during aging

- Sleep/activity rhythms decay in aging while clock gene oscillations persist in central clock

- Clock mutants with disrupted rhythms show accelerated aging phenotypes 
Clock mechanism

Young
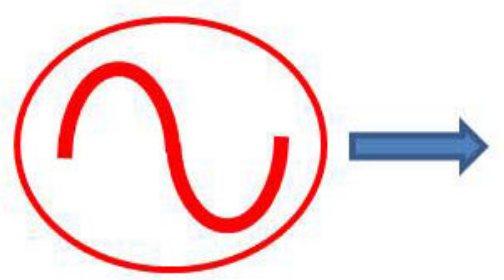

Old
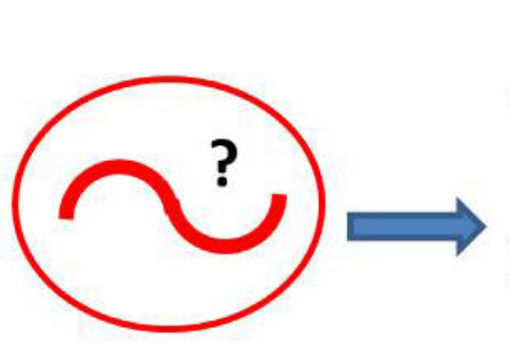

?

Clock mutant

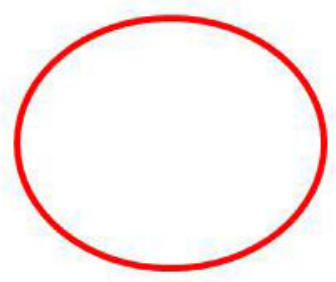

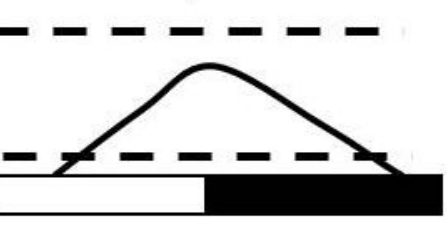
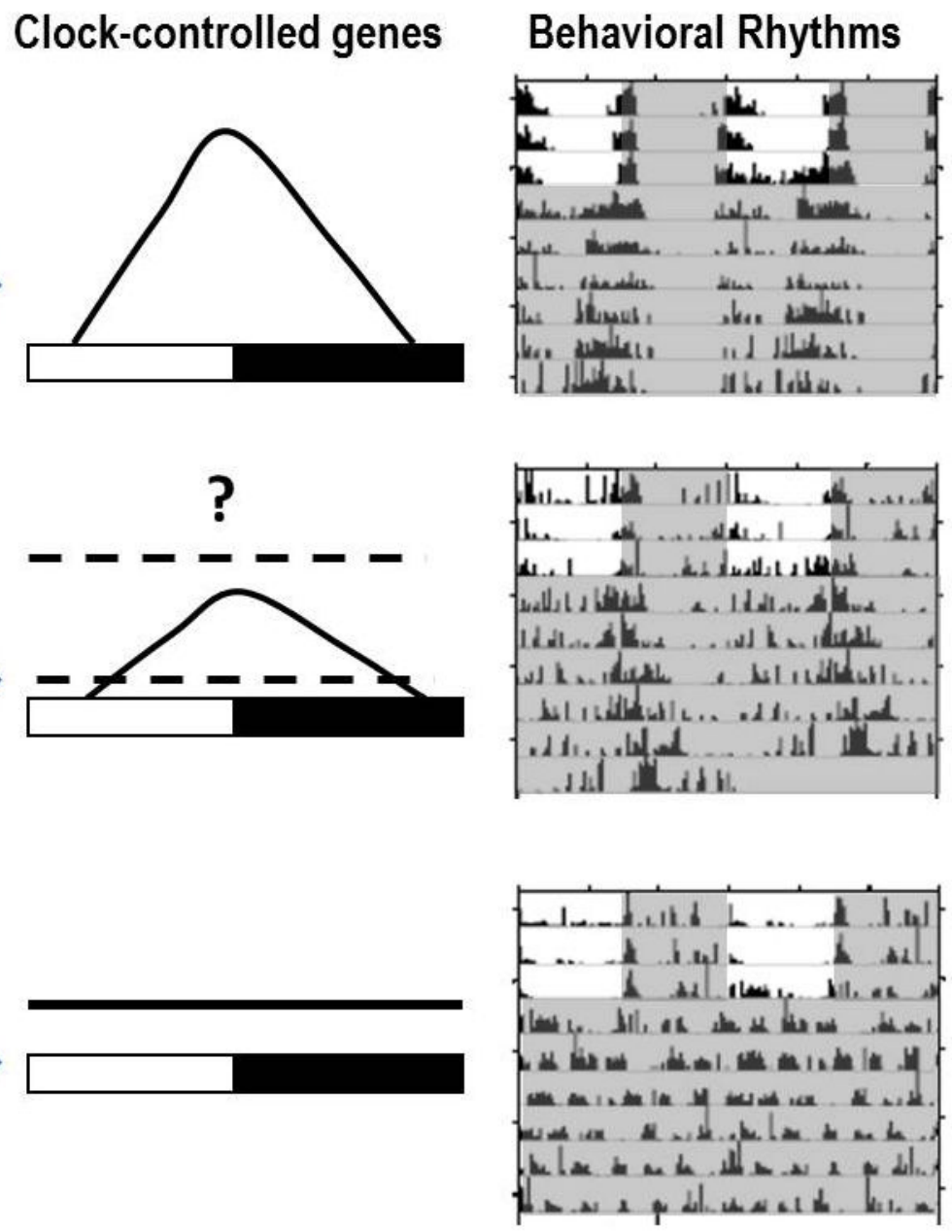

Figure 1.

Scheme summarizing changes in the circadian system of D. melanogaster. Clock oscillations that are strong in young flies, may be dampened in old flies, and are gone altogether in clock mutant flies. Many output rhythms are governed by the clock-controlled genes (CCGs) in young flies. It is not known how expression profiles of CCGs change during aging: the rhythms may become attenuated, or abolished at the peak or through (indicated by the broken lines). Arrhythmic flies with null mutation in per or cyc gene show accelerated aging and shorter lifespan, respectively. 